\title{
Genotype-phenotype correlation in migraine without aura focusing on the rs1835740 variant on 8q22.1
}

\author{
Anne Francke Christensen • Han Le • \\ Malene Kirchmann • Jes Olesen
}

Received: 3 August 2011/Accepted: 2 September 2011/Published online: 1 October 2011

(C) The Author(s) 2011. This article is published with open access at Springerlink.com

\begin{abstract}
A large two-stage GWAS by Antilla et al. reported the minor allele of rs 1835740 on $8 \mathrm{q} 22.1$ to be associated with common types of migraine. The objective of the present study was to determine the clinical correlate of the variant in migraine without aura (MO). Clinical data on 339 successfully genotyped MO patients (patients with attacks of migraine without aura and no attacks of migraine with aura) were obtained by an extensive validated semistructured telephone interview performed by a physician or a trained senior medical student. Reliable, systematic and extensive data on symptoms, age of onset, attack frequencies and duration, relevant comorbidity, specific provoking factors including different hormonal factors in females, and effect and use of medication, both abortive and prophylactic, were thereby obtained. A comparison of carriers and non-carriers were performed. Comparison of homozygotes with heterozygotes was not performed as the number of homozygotes was too small for statistical purposes. Data from other MO populations in the GWAS by Antilla et al. were not included as phenotype and clinical data were obtained differently. While thousands of patients are needed to detect a genetic variant like rs1835740, 339 are sufficient to detect meaningful clinical differences. 136 of 339 patients were carriers of the variant, 15 were homozygous. Comparison of carriers with non-carriers showed no significant difference in any of the parameters studied. In conclusion, the rs 1835740 variant has no significant influence on the clinical expression of MO.
\end{abstract}

A. F. Christensen · H. Le · M. Kirchmann · J. Olesen ( $\square)$ Department of Neurology, Danish Headache Center, University of Copenhagen, Glostrup University Hospital, Nordre Ringvej 57, Glostrup, 2600 Copenhagen, Denmark e-mail: jeol@glo.regionh.dk
Keywords Migraine - Migraine without aura · Phenotype - Genotype $\cdot$ Clinical characteristics

\section{Introduction}

Genetic factors have been demonstrated to play an important role in the pathogenesis of migraine. The rare familial hemiplegic migraine (FHM), defined by the presence of a transient hemiplegia during the aura phase, is dominantly inherited with at least four different genetic subtypes [1]. In contrast, migraine without aura (MO) and migraine with typical aura (MA) have multifactorial inheritance [2-7]. The co-occurrence of MO and MA in monozygotic and dizygotic twin pairs is not higher than expected by chance, which together with the different epidemiology and clinical features of the two indicates that MO and MA are distinct disorders with some clinical and etiological overlap $[5,8]$. This study will concentrate on MO. The influence of a genetic factor in MO is supported by a twofold increased risk of MO in first degree relatives of probands with $\mathrm{MO}$ compared to the general population [3]. Furthermore, the concordance rate of MO in monozygotic twin pairs is significantly higher than in dizygotic twin pairs (28 vs. 18\%) [9], and the heritability has been estimated to $50 \%$ [2].

Until recently, no reproducible genetic association or linkage has been reported in MO. However, a large two stage genome-wide association study (GWAS) by Antilla et al. reported the minor allele of rs 1835740 on chromosome 8q22.1 to be associated with migraine with an overall meta-analysis $p$ value of $1.69 \times 10^{-11}$ [10]. The study comprised case materials from five European countries, 2,731 migraine cases were included in the discovery stage and 3,202 in the replication stage. The allele was found to 
be overrepresented in all of the following groups: all migraine patients, MA patients without attacks of MO (MA only), patients with attacks of both MA and MO, and MO patients without attacks of MA (MO only).

The clinical correlate of this variant, if any, remains to be determined. In our genetic studies, we have systematically collected extensive clinical data using a semistructured telephone interview by a physician. We are thus able to report on the phenotype-genotype correlation based on detailed clinical information in 339 patients with MO and no attacks of MA from the Danish subsample. The aim of the present study was thus to compare clinical data in MO patients with and without the rs 1835740 variant.

We have not attempted to include the other groups from the two-stage GWAS in this analysis in that the phenotyping has been done differently and with different elaboration on clinical features from group to group. Thus, it was not possible to pool clinical data. If the difference in clinical features between patients with and without the variant is of any clinical importance, 339 patients should be enough for this purpose.

\section{Methods}

Ascertainment of MO patients without attacks of MA (MO only)

Patients having a MO diagnosis living in the eastern part of Denmark were extracted from case files at the Danish Headache Center. The recruited patients, all ethnical Caucasians, received a posted letter stating that the objective of the survey was to study the inheritance of migraine and would involve a telephone interview and a blood sample. They were asked to return a slip in a prepaid envelope confirming whether they agreed to participate. 606 patients were recruited and 407 agreed to participate, corresponding to a response rate of $67.4 \%$. These patients were then contacted for an extensive semi-structured telephone interview by a physician or a trained senior medical student. The validated semi-structured telephone interview was based on the diagnostic criteria of The International Headache Society (IHS), and the diagnosis was given according to the IHS 2004 criteria [11, 12]. A total of 360 patients were given the diagnosis MO. Of these, 56 were excluded because of co-occurrence of MA. Thus, 304 were given the diagnosis 'MO only' and included. In addition, 81 MO-only patients were diagnosed and included from another study recruiting MA families using the same semistructured telephone interview [13]. The total number of MO-only patients was thus 385 . A blood sample was collected from each of the 385 MO-only patients, and 340 were successfully genotyped. One was hereafter excluded due to missing data; hence, the analysis in this study was based on data in 339 MO-only patients. All subjects provided written informed consent. The project was approved by the Danish Ethics Committees (application no. KA 94076m).

\section{The two-stage GWAS}

The method is fully published by Antilla et al. A short description is given here to facilitate the understanding of our work.

In the discovery stage, a clinic-based sample of 3,279 migraineurs from Finland, Germany and The Netherlands was studied and genotyped at the Wellcome Trust Sanger Institute using Illumina (610K and $550 \mathrm{~K})$ SNP microarrays against 10,747 population-matched controls [10]. In the replication stage, a further 3,202 migraineurs from Iceland (using Illumina Human Hap $317 \mathrm{~K}, 370 \mathrm{~K}, 610 \mathrm{~K}$ or $1 \mathrm{M}$ bead arrays at deCODE genetics), Denmark (using Centaurus platform [Nanogen inc.] at deCODE genetics), The Netherlands (TaqMan technology [Applied Biosystems, Life] at Leiden University Medical Center) and Germany (Illumina HumanHap $610 \mathrm{~K}$ array at the Institute of Human Genetics at the Helmholtz Zentrum, Munich) were studied against 40,062 population-matched controls.

The diagnoses were given by headache experts using a combination of questionnaires and individual interviews based on the IHS 2004 criteria [11]. The following diagnostic subgroups were analyzed: (1) all migraine patients ('all migraine'), (2) MA patients without attacks of MO ('MA only'), (3) patients with attacks of both MA and MO ('both MA and MO'), and (4) MO patients without attacks of MA ('MO only').

For the meta-analysis of discovery and replication samples the Cochran-Mantel-Haenszel (CMH) association analysis with a significance threshold of $p \leq 5 \times 10^{-8}$ was used. In the discovery sample, 2,731 cases and 10,747 controls passed quality control steps, and 429,912 markers were successfully genotyped. Only one marker, rs 1835740 on chromosome 8q22.1, showed significant association with migraine in the multi-population $\mathrm{CMH}$ analysis. The minor allele (A) of marker rs1835740 was associated with 'all migraine' with $p=5.38 \times 10^{-9}$ and odds ratios ranging between 1.21 and 1.33 . The result was confirmed in the replication study with a final $p=1.69 \times 10^{-11}$ in the $\mathrm{CMH}$ meta-analysis for all migraine sample together.

In the HapMap Phase II data [14], marker rs1835740 is located between the two potentially interesting genes, MTDH and PCGP, which are both involved in glutamate homeostasis. The effect of the marker on gene expression was analyzed in human fibroblasts, primary $\mathrm{T}$ cells and lymphoblastoid cell lines obtained from umbilical cords. 
The risk allele A of rs 1835740 was found to have significant correlation to higher MTDH expression in lymphoblastoid cell lines.

Statistical methods

All data were processed and analyzed using Statistical Package for the Social Sciences (SPSS) version 18.0 in Windows 7.0. Two-tailed Student's $t$ test and one-way ANOVA was used to compare means for numerical data. $\chi^{2}$ test was used to compare categorical data.

A comparison of carriers of the rs 1835740 variant with non-carriers was performed. Thereafter carriers were subdivided into homo- and heterozygotes. No statistical tests were performed on homozygotes versus heterozygotes as the sample of homozygotes was too small. Finally, a subanalysis in females was done. Sub-analysis in males was not performed as this sub-group was too small for a meaningful analysis.

\section{Results}

136 out of $339(40 \%)$ apparently non-related MO probands were carriers of the rs 1835740 variant, hereof 20 out of 47 males and 116 out of 292 females. Of the carriers, 15 were homozygous ( 1 male and 14 females) and 121 were heterozygous (19 males and 102 females). In carriers, the average age was $43.8 \pm 12.8$, and in non-carriers $43.6 \pm$ $11.5, p=0.880$. The average age of onset in carriers was $20.9 \pm 12.0$, and in non-carriers $20.9 \pm 10.6, p=0.995$. The average duration of migraine was in the interval $4-23 \mathrm{~h}$ in $37.5 \%$ of carriers and in $36.9 \%$ of non-carriers, and 1-3 days in $54.4 \%$ of carriers and in $56.7 \%$ in noncarriers. Thus, carriers and non-carriers were comparable in average age, age of onset and average duration of migraine. Attack frequencies through life and during the past year were also evenly distributed for carriers and noncarriers (data not shown).

Table 1 summarizes the migraine-specific symptoms of carriers versus non-carriers with corresponding $p$ values, and of homo- and heterozygotes, respectively. Table 2 describes the comorbidity. Provoking factors are described in Table 3, hormonal factors in females in Table 4. The effect and use of medicine is presented in Table 5.

No statistically significant association with the A-allele of rs 1835740 was found in the comparison of carriers with non-carriers for any of the parameters studied. However, when carriers were divided in homo- and heterozygotes and compared to non-carriers, a tendency that heterozygotes were comparable to non-carriers, and that homozygotes tended to differ in a non-significant manner from these two other groups, could be observed.
A sub-analysis in females (not shown) gave the same results as in the whole material. No statistical calculations were done in males because of their small number. Numerically, males seemed to follow the general picture.

\section{Discussion}

No statistically significant association with the A-allele rs1835740 was found for any of the parameters that were studied: symptoms, comorbidity, provoking factors or the effect and use of different medical treatment. However, while heterozygotes were comparable to non-carriers, homozygotes tended to differ in a non-significant manner in some parameters. Homozygosity for the A-allele of rs1835740 might thus have a small influence on the phenotype of MO, but it could easily be a false trend because of the small number of homozygotes. The sub-analysis in females did not show any difference from the overall analysis, and the male sample was too small for statistical calculation. Thus, the present study suggests that the rs1835740 variant has no influence on the clinical expression of MO.

Why study MO as a separate entity?

It has been questioned whether MO and MA are two distinct disorders or a single entity $[15,16]$, but considerable evidence supports the former. All together, the different epidemiology, the different clinical features, difference in comorbidity, the presence or absence of measurable cortical spreading depression, different provoking factors, different effect of different preventive medicine and twin studies showing lower concordance-rate in MO than in $\mathrm{MA}$, indicate that MO and MA are distinct disorders with some clinical and etiological overlap [5, 8, 17-20]. Therefore, the present study concentrated on MO only.

The original large study by Antilla et al. showed consistently stronger association with the presence of the rs1835740 A-allele for MA-only groups [10]. The present study indicates that the A-allele of rs 1835740 probably has no crucial influence on the phenotype of MO. Maybe studies of clinical features in MA will reveal a more clear difference according to the presence or absence of rs 1835740 A-allele in MA patients. This would further support the assumption that $\mathrm{MO}$ and MA are distinct disorders.

Methodological considerations

In the absence of a biochemical marker or paraclinical test, the diagnosis of migraine is purely clinical. The differential diagnosis between tension-type headache (TTH) and migraine is often difficult, and also the differentiation 
Table 1 Symptoms associated with attacks of migraine in 'migraine without aura' patients divided in subgroups: carriers and non-carriers of the rs 1835740 variant, and homo- and heterozygotes of the rs1835740 variant

\begin{tabular}{|c|c|c|c|c|c|c|}
\hline Symptoms & $\begin{array}{l}\text { Carriers } \\
\%(n)\end{array}$ & $\begin{array}{l}\text { Non-carriers } \\
\%(n)\end{array}$ & $p^{*}$ & $\begin{array}{l}\text { Homo } \\
\%(n)\end{array}$ & $\begin{array}{l}\text { Hetero } \\
\%(n)\end{array}$ & $\begin{array}{l}\text { Total number } \\
\text { of answers }\end{array}$ \\
\hline Unilateral location & $83.9(104)$ & 87.7 (164) & 0.338 & $80.0(12)$ & $84.4(92)$ & 311 \\
\hline Pulsating quality & $81.0(102)$ & $82.6(152)$ & 0.710 & $71.4(10)$ & $82.1(92)$ & 310 \\
\hline Moderate or severe pain intensity & $100.0(136)$ & $100.0(203)$ & - & $100.0(15)$ & $100.0(121)$ & 339 \\
\hline Aggravation by physical activity & $91.0(121)$ & $93.0(186)$ & 0.500 & $80.0(12)$ & $92.4(109)$ & 333 \\
\hline Nausea & $93.2(124)$ & $94.5(188)$ & 0.642 & $93.3(14)$ & $93.2(110)$ & 332 \\
\hline Vomiting & $67.7(86)$ & 69.4 (127) & 0.753 & $76.9(10)$ & $66.7(76)$ & 310 \\
\hline Photophobia & $92.3(120)$ & $90.2(175)$ & 0.516 & $100.0(14)$ & $91.4(106)$ & 324 \\
\hline Phonophobia & $88.4(114)$ & $82.7(158)$ & 0.165 & $100.0(14)$ & $87.0(100)$ & 320 \\
\hline Osmophobia & $52.2(59)$ & $57.2(97)$ & 0.422 & $53.8(7)$ & $52.0(52)$ & 283 \\
\hline
\end{tabular}

* $p$ values for comparison of carriers and non-carriers

Table 2 Comorbidity in 'migraine without aura' patients divided in subgroups: carriers and non-carriers of the rs 1835740 variant, and homoand heterozygotes of the rs1835740 variant

\begin{tabular}{|c|c|c|c|c|c|c|}
\hline Comorbidity & $\begin{array}{l}\text { Carriers } \\
\%(n)\end{array}$ & $\begin{array}{l}\text { Non-carriers } \\
\%(n)\end{array}$ & $p^{*}$ & $\begin{array}{l}\text { Homo } \\
\%(n)\end{array}$ & $\begin{array}{l}\text { Hetero } \\
\%(n)\end{array}$ & $\begin{array}{l}\text { Total number } \\
\text { of answers }\end{array}$ \\
\hline Comotio & $44.9(61)$ & $41.0(82)$ & 0.483 & $33.3(5)$ & $46.3(56)$ & 336 \\
\hline Cranial fracture & $1.5(2)$ & $1.0(2)$ & 0.696 & $0.0(0)$ & $1.7(2)$ & 336 \\
\hline Encephalitis & $0.0(0)$ & $0.5(1)$ & 0.409 & $0.0(0)$ & $0.0(0)$ & 336 \\
\hline Meningitis & $1.5(2)$ & $2.0(4)$ & 0.719 & $6.7(1)$ & $0.8(1)$ & 336 \\
\hline Cerebral thrombosis & $0.7(1)$ & $0.0(0)$ & 0.225 & $0.0(0)$ & $0.8(1)$ & 336 \\
\hline Cerebral hemorrhage & $0.0(0)$ & $0.0(0)$ & _- & $0.0(0)$ & $0.0(0)$ & 336 \\
\hline Transient cerebral ischemia (TCI) & $0.0(0)$ & $0.0(0)$ & - & $0.0(0)$ & $0.0(0)$ & 336 \\
\hline Arterial hypertension & $10.3(14)$ & $16.5(33)$ & 0.107 & $0.0(0)$ & $11.6(14)$ & 336 \\
\hline Tension type headache (TTH) & $65.4(89)$ & $73.6(147)$ & 0.106 & 86.7 (13) & $62.8(76)$ & 337 \\
\hline
\end{tabular}

* $p$ values for comparison of carriers and non-carriers

Table 3 Migraine provoking factors in 'migraine without aura' patients divided in subgroups: carriers and non-carriers of the rs 1835740 variant, and homo- and heterozygotes of the rs 1835740 variant

\begin{tabular}{|c|c|c|c|c|c|c|}
\hline Provoking factors & $\begin{array}{l}\text { Carriers } \\
\%(n)\end{array}$ & $\begin{array}{l}\text { Non-carriers } \\
\%(n)\end{array}$ & $p^{*}$ & $\begin{array}{l}\text { Homo } \\
\%(n)\end{array}$ & $\begin{array}{l}\text { Hetero } \\
\%(n)\end{array}$ & $\begin{array}{l}\text { Total number } \\
\text { of answers }\end{array}$ \\
\hline Attacks provokable & $26.6(29)$ & $21.7(26)$ & 0.348 & $9.1(1)$ & $28.6(28)$ & 275 \\
\hline Physical activity & $32.7(37)$ & $30.2(51)$ & 0.649 & $8.3(1)$ & $35.6(36)$ & 282 \\
\hline Stress & $69.3(79)$ & $69.8(120)$ & 0.933 & $61.5(8)$ & $70.3(71)$ & 286 \\
\hline Weekend/holiday & $49.6(56)$ & $51.5(88)$ & 0.753 & $53.8(7)$ & $49.0(49)$ & 284 \\
\hline Food & $50.4(57)$ & $41.2(70)$ & 0.125 & $61.5(8)$ & $49.0(49)$ & 283 \\
\hline Alcohol & $26.4(29)$ & $19.2(32)$ & 0.157 & $7.7(1)$ & $28.9(28)$ & 277 \\
\hline Hormonal factors, females only: menstrual migraine ${ }^{a}$ & $51.5(50)$ & $56.7(85)$ & 0.430 & $41.7(5)$ & $52.9(45)$ & 247 \\
\hline
\end{tabular}

* $p$ values for comparison of carriers and non-carriers

a Attacks associated to menstruation

between MO and MA is sometimes uncertain. We used an extensive, validated, semi-structured telephone interview performed by a physician or a trained senior medical student [12]. Thus, our clinical data are reliable as well as systematic and extensive.
The sample of MO patients in this study was recruited from a specialized clinical sample and thus represents a fairly severely affected group. We cannot exclude phenotypic differences between those with and without the variant in a population of less affected individuals. 
Table 4 Effect of hormonal factors on migraine attack frequency, females only, in 'migraine without aura' patients divided in subgroups: carriers and non-carriers of the rs1835740 variant, and homo- and heterozygotes of the rs1835740 variant

\begin{tabular}{|c|c|c|c|c|c|}
\hline Attack frequency & Unchanged & Higher & Lower & Irrelevant & Total number of answers \\
\hline During pregnancy & & & & & 252 \\
\hline Non-carriers & $7.8(12)$ & $5.2(8)$ & $48.4(74)$ & $38.6(59)$ & \\
\hline Hetero & $11.5(10)$ & $2.3(2)$ & $40.2(35)$ & $46.0(40)$ & \\
\hline Homo & $0.0(0)$ & $0.0(0)$ & $41.7(5)$ & $58.3(7)$ & \\
\hline Using birth-control pills & & & & & 246 \\
\hline Non-carriers & $38.0(57)$ & $20.0(30)$ & $5.3(8)$ & $36.7(55)$ & \\
\hline Hetero & $47.1(40)$ & $9.4(8)$ & $4.7(4)$ & $38.8(33)$ & \\
\hline Homo & $36.4(4)$ & $9.1(1)$ & $9.1(1)$ & $45.5(5)$ & \\
\hline After meno pause & & & & & 245 \\
\hline Non-carriers & $10.8(16)$ & $7.4(11)$ & $6.8(10)$ & $75.0(111)$ & \\
\hline Hetero & $15.3(13)$ & $10.6(9)$ & $2.4(2)$ & $71.8(61)$ & \\
\hline Homo & $0.0(0)$ & $16.7(2)$ & $25.0(3)$ & $58.3(7)$ & \\
\hline
\end{tabular}

Table 5 Effect and use of medicine in 'migraine without aura' patients divided in subgroups: carriers and non-carriers of the rs1835740 variant, and homo- and heterozygotes of the rs 1835740 variant

\begin{tabular}{|c|c|c|c|c|c|c|}
\hline Effect and use of medicine & $\begin{array}{l}\text { Carriers } \\
\%(n)\end{array}$ & $\begin{array}{l}\text { Non-carriers } \\
\%(n)\end{array}$ & $p^{*}$ & $\begin{array}{l}\text { Homo } \\
\%(n)\end{array}$ & $\begin{array}{l}\text { Hetero } \\
\%(n)\end{array}$ & $\begin{array}{l}\text { Total number } \\
\text { of answers }\end{array}$ \\
\hline Effect of triptans & $83.2(94)$ & $85.8(145)$ & 0.601 & $84.6(11)$ & $83.0(83)$ & 282 \\
\hline Effect of prophylactic medication & $33.0(37)$ & $28.5(49)$ & 0.582 & $46.2(6)$ & $31.3(31)$ & 284 \\
\hline Under current prophylactic medication & $35.4(40)$ & $32.0(54)$ & 0.548 & $23.1(3)$ & $37.0(37)$ & 282 \\
\hline Other current daily medication & $36.0(41)$ & $35.1(60)$ & 0.879 & $23.1(3)$ & $37.6(38)$ & 285 \\
\hline Previous/current treatment of med. overuse headache & $32.1(36)$ & $29.2(50)$ & 0.604 & $23.1(3)$ & $33.3(33)$ & 283 \\
\hline
\end{tabular}

* $p$ values for comparison of carriers and non-carriers

The number of patients in this study was 339. This is usually an adequate number for phenotype-genotype correlation studies and sufficient for most purposes. Thousands of probands are needed to find a genetic association like the rs1835740 variant. However, if thousands of patients are needed to show significance of a difference of a certain clinical feature between two groups, this difference will not be of clinical importance. For analysis in relatively small groups, e.g., males, our material is insufficient. Most importantly, the group of homozygotes was far too small for a meaningful statistical analysis. It seems that if any phenotypical correlate of the variant exists, it must be found in this group. However, a very large material would be necessary to get enough homozygous patients.

\section{Migraine and glutamate}

Marker rs 1835740 is located between the two potentially interesting genes, MTDH and PCGP, which are both involved in glutamate homeostasis. The rs 1835740 genotype was found by Antilla et al. to be significantly correlated to MTDH expression in lymphoblastoid cell lines [10]. In astrocytes, MTDH has been shown to downregulate GLT-1, the gene encoding the major glutamate transporter. The new genetic variant may thus contribute to the understanding of the role of glutamate in migraine. Glutamate is involved in central sensitization which is considered to be a crucial part of migraine pathophysiology [21], and glutamate accumulation increases the susceptibility to cortical spreading depression $[22,23]$.

Glutamate is the major excitatory neurotransmitter in the CNS and therefore plays a crucial role in the mediation of excitatory synaptic transmission [24]. The anatomic structures involved in the migraine pain pathway, including the trigeminal ganglion (TG), the trigemino-cervical complex (TCC) and thalamus, contain glutamate-positive neurons $[25,26]$. Glutamate exhibits its actions through activation of ionotropic and metabotropic receptors (GluRs), and the pharmacological distinction of these is well documented [27]. Glutamate is released from the TCC in response to stimulation of dural structures. In TG neurons, glutamate is released along with calcitonin generelated peptide (CGRP), and the majority of glutamatergic neurons in TG carry $5-\mathrm{HT}_{1 \mathrm{~B} / \mathrm{D} / \mathrm{F}}$ receptors, which could possibly modulate glutamate release [28]. Glutamate also 
plays a significant role in the transmission of nociceptive information in the sensory thalamus $[29,30]$.

Migraineurs have elevated levels of glutamate and glutamine in the cerebrospinal fluid compared with controls, and a positive correlation between glutamate levels and mean headache scores has been reported [31-33]. Although the variant only explains a small fraction of the overall genetic variance, it increases the interest in glutamatergic mechanisms in migraine.

\section{Personalized medicine and future perspectives}

There is still an unmet need to find more effective, tolerable and safe treatments for migraine, especially regarding preventive agents. MO is clinically well defined as a syndrome, but it has not been possible to divide MO into subtypes based on clinical features. The tolerability, safety and efficacy of each type of preventive medicine are individual for every patient and not predictable. Thus, 'trial and error' is still the only possible treatment strategy. If a certain genotype was associated with certain clinical features, it would perhaps be possible to select patients for treatment based on clinical criteria. Unfortunately, the present study did not reveal a significant difference in clinical features according to the presence or absence of the rs 1835740 variant. Another approach would be to compare the effect of drugs in patients with and without the variant. Especially, the effects of glutamate-modulating agents would be interesting to investigate. If this strategy gives results and as the technology of genotyping gets more economic and accessible, a future perspective could be to select patients for treatment based on genotyping [34].

Acknowledgments We wish to thank all individuals in our cohort for their participation. Especially, thanks to our collaborators at deCODE genetics, Iceland, who performed the genotyping in this study. Antilla et al. did the meta-analysis resulting in the finding of the susceptibility variant rs1835740 making this study possible.

\section{Conflict of interest None.}

Open Access This article is distributed under the terms of the Creative Commons Attribution License which permits any use, distribution and reproduction in any medium, provided the original author(s) and source are credited.

\section{References}

1. de VB, Frants RR, Ferrari MD, van den Maagdenberg AM (2009) Molecular genetics of migraine. Hum Genet 126(1):115-132

2. Gervil M, Ulrich V, Kaprio J, Olesen J, Russell MB (1999) The relative role of genetic and environmental factors in migraine without aura. Neurology 53(5):995-999

3. Russell MB, Olesen J (1995) Increased familial risk and evidence of genetic factor in migraine. BMJ 311(7004):541-544
4. Russell MB, Iselius L, Olesen J (1995) Inheritance of migraine investigated by complex segregation analysis. Hum Genet 96(6):726-730

5. Russell MB, Rasmussen BK, Fenger K, Olesen J (1996) Migraine without aura and migraine with aura are distinct clinical entities: a study of four hundred and eighty-four male and female migraineurs from the general population. Cephalalgia 16(4):239-245

6. Ulrich V, Gervil M, Kyvik KO, Olesen J, Russell MB (1999) Evidence of a genetic factor in migraine with aura: a populationbased Danish twin study. Ann Neurol 45(2):242-246

7. Ulrich V, Gervil M, Kyvik KO, Olesen J, Russell MB (1999) The inheritance of migraine with aura estimated by means of structural equation modelling. J Med Genet 36(3):225-227

8. Russell MB, Ulrich V, Gervil M, Olesen J (2002) Migraine without aura and migraine with aura are distinct disorders. A population-based twin survey. Headache 42(5):332-336

9. Gervil M, Ulrich V, Kyvik KO, Olesen J, Russell MB (1999) Migraine without aura: a population-based twin study. Ann Neurol 46(4):606-611

10. Anttila V, Stefansson H, Kallela M, Todt U, Terwindt GM, Calafato MS et al (2010) Genome-wide association study of migraine implicates a common susceptibility variant on $8 \mathrm{q} 22.1$. Nat Genet

11. Headache Classification Committee of the International Headache Society (2004) The international classification of headache disorders: 2nd edition. Cephalalgia 24 Suppl 1:9-160

12. Russell MB, Rasmussen BK, Thorvaldsen P, Olesen J (1995) Prevalence and sex-ratio of the subtypes of migraine. Int J Epidemiol 24(3):612-618

13. Eriksen MK, Thomsen LL, Andersen I, Nazim F, Olesen J (2004) Clinical characteristics of 362 patients with familial migraine with aura. Cephalalgia 24(7):564-575

14. (2011) http://www.hapmap.ncbi.nlm.nih.gov/

15. Ligthart L, Boomsma DI, Martin NG, Stubbe JH, Nyholt DR (2006) Migraine with aura and migraine without aura are not distinct entities: further evidence from a large Dutch population study. Twin Res Hum Genet 9(1):54-63

16. Nyholt DR, Gillespie NG, Heath AC, Merikangas KR, Duffy DL, Martin NG (2004) Latent class and genetic analysis does not support migraine with aura and migraine without aura as separate entities. Genet Epidemiol 26(3):231-244

17. Chronicle EP, Pearson AJ, Mulleners WM (2006) Objective assessment of cortical excitability in migraine with and without aura. Cephalalgia 26(7):801-808

18. Manzoni GC, Torelli P (2008) Migraine with and without aura: a single entity? Neurol Sci 29(Suppl 1):S40-S43

19. Olesen J, Larsen B, Lauritzen M (1981) Focal hyperemia followed by spreading oligemia and impaired activation of $\mathrm{rCBF}$ in classic migraine. Ann Neurol 9(4):344-352

20. Olesen J, Tfelt-Hansen P, Henriksen L, Larsen B (1981) The common migraine attack may not be initiated by cerebral ischaemia. Lancet 2(8244):438-440

21. Goadsby PJ (2005) Migraine pathophysiology. Headache 45(Suppl 1):S14-S24

22. Lauritzen M (1994) Pathophysiology of the migraine aura. The spreading depression theory. Brain 117(Pt 1):199-210

23. Hadjikhani N, Sanchez Del RM, Wu O, Schwartz D, Bakker D, Fischl B et al (2001) Mechanisms of migraine aura revealed by functional MRI in human visual cortex. Proc Natl Acad Sci USA 98(8):4687-4692

24. Andreou AP, Goadsby PJ (2009) Therapeutic potential of novel glutamate receptor antagonists in migraine. Expert Opin Invest Drugs 18(6):789-803

25. Greenamyre JT, Young AB, Penney JB (1984) Quantitative autoradiographic distribution of $\mathrm{L}$-[3H]glutamate-binding sites in rat central nervous system. J Neurosci 4(8):2133-2144 
26. Kai-Kai MA, Howe R (1991) Glutamate-immunoreactivity in the trigeminal and dorsal root ganglia, and intraspinal neurons and fibres in the dorsal horn of the rat. Histochem J 23(4):171-179

27. Kew JN, Kemp JA (2005) Ionotropic and metabotropic glutamate receptor structure and pharmacology. Psychopharmacology 179(1):4-29

28. Ma QP (2001) Co-localization of 5-HT(1B/1D/1F) receptors and glutamate in trigeminal ganglia in rats. Neuroreport 12(8):15891591

29. Salt TE (2002) Glutamate receptor functions in sensory relay in the thalamus. Phil Trans R Soc Lond B Biol Sci 357(1428):1759_ 1766

30. Silva E, Quinones B, Freund N, Gonzalez LE, Hernandez L (2001) Extracellular glutamate, aspartate and arginine increase in the ventral posterolateral thalamic nucleus during nociceptive stimulation. Brain Res 923(1-2):45-49

31. Martinez F, Castillo J, Rodriguez JR, Leira R, Noya M (1993) Neuroexcitatory amino acid levels in plasma and cerebrospinal fluid during migraine attacks. Cephalalgia 13(2):89-93

32. Peres MF, Zukerman E, Senne Soares CA, Alonso EO, Santos BF, Faulhaber MH (2004) Cerebrospinal fluid glutamate levels in chronic migraine. Cephalalgia 24(9):735-739

33. Rothrock JF, Mar KR, Yaksh TL, Golbeck A, Moore AC (1995) Cerebrospinal fluid analyses in migraine patients and controls. Cephalalgia 15(6):489-493

34. Simmaco M, Borro M, Missori S, Martelletti P (2009) Pharmacogenomics in migraine: catching biomarkers for a predictable disease control. Expert Rev Neurother 9(9):1267-1269 